\title{
Evidence, Mechanism, and Clinical Relevance of the Transdifferentiation from Lung Adenocarcinoma to Squamous Cell Carcinoma
}

\author{
Shenda Hou, ${ }^{\dagger \ddagger}$ Shiyu Zhou, ${ }^{* \dagger \neq}$ Zhen Qin, ${ }^{* \dagger \ddagger 8}$ Liu Yang, ${ }^{\dagger \dagger}$ Xiangkun Han, ${ }^{\star \dagger \ddagger}$ Shun Yao, ${ }^{* \dagger \ddagger}$ and \\ Hongbin $\mathrm{Ji}^{* \dagger+\uparrow}$
}

From the Chinese Academy of Sciences Key Laboratory of Systems Biology, * the Chinese Academy of Sciences Center for Excellence in Molecular Cell Science, ${ }^{\dagger}$ and the Innovation Center for Cell Signaling Network, ${ }^{\ddagger}$ Institute of Biochemistry and Cell Biology, Shanghai Institutes for Biological Sciences, Chinese Academy of Sciences, Shanghai; the University of Chinese Academy of Sciences, ${ }^{\S}$ Beijing; and the School of Life Science and Technology, ${ }^{\circledR}$ Shanghai Tech University, Shanghai, China

\author{
Accepted for publication \\ January 19, 2017. \\ Address correspondence to \\ Hongbin Ji, Ph.D., Institute of \\ Biochemistry and Cell \\ Biology, Shanghai Institutes \\ for Biological Sciences, \\ Chinese Academy of Sciences, \\ 320 Yueyang Road, Shanghai, \\ China 200031. E-mail: hbji@ \\ sibcb.ac.cn.
}

\begin{abstract}
Lung adenocarcinoma (ADC) and squamous cell carcinoma (SCC) are two distinct subtypes of nonsmall-cell lung carcinoma. Interestingly, approximately $4 \%$ to $9 \%$ of human non-small-cell lung carcinoma tumors contain mixed adenomatous and squamous pathologies in a single lesion, clinically termed adenosquamous cell carcinoma. More important, these two different pathological components frequently share identical oncogenic mutations, indicative of a potential transition. Indeed, recent data have provided convincing evidence in supporting the $A D C$ to SCC transdifferentiation in lungs. In the liver kinase B1 (official name STK11)-deficient mouse model, lung ADC can progressively transdifferentiate to SCC through pathologically mixed adenosquamous cell carcinoma as the intermediate status. Mechanistic studies further identify essential roles of extracellular matrix remodeling and metabolic reprogramming during this phenotypic transition. Small molecular compounds, including lysyl oxidase inhibitors and reactive oxygen species-inducing reagents such as phenformin, significantly accelerate the transition from lung $A D C$ to $S C C$ and thus confer lung tumors with drug resistance. Consistent with these findings, recent clinical studies have shown that epidermal growth factor receptormutant lung $A D C$ can transdifferentiate to SCC in relapsed cancer patients. Together, these data support that this phenotypic transition from lung $A D C$ to SCC might represent a novel mechanism for drug resistance. This review will summarize our current understanding of the transdifferentiation from lung ADC to SCC. (Am J Pathol 2017, 187: 954-962; http://dx.doi.org/10.1016/j.ajpath.2017.01.009)
\end{abstract}

Lung cancer is one of the most deadly diseases worldwide, with a 5-year survival rate at approximately $15 \% .{ }^{1}$ Nonsmall-cell lung carcinoma (NSCLC) is the major subtype of lung cancer, which can be further divided into three subtypes, including lung adenocarcinoma (ADC), squamous cell carcinoma (SCC), and large-cell carcinoma. Lung ADC and SCC, which account for $>85 \%$ of NSCLC, harbor different features (Table 1). First, most lung ADCs are considered to originate from type II pneumocytes or club cells, and maintain glandular differentiation with acini, tubules, or papillary structures. ${ }^{2}$ In contrast, lung SCCs are derived from basal cells located underneath trachea or bronchus epithelium ${ }^{3}$ and exhibit unique characteristic of keratin or intracellular bridges. ${ }^{2}$ Second, lung ADCs are frequently located at distal bronchioles, whereas SCCs are often found at more proximal airways. Moreover, lung ADCs and SCCs show distinct gene expression profiles as

\footnotetext{
Supported by the Strategic Priority Research Program of the Chinese Academy of Sciences grant XDB19000000 (H.J.); the National Natural Science Foundation of China grants 31621003 (H.J.), 31370747 (H.J.), 81325015 (H.J.), 81430066 (H.J.), and 81402276 (X.H.); and Science and Technology Commission of Shanghai Municipality grant 15XD1504000 (H.J. and X.H.).

S.H. and S.Z. contributed equally to this work.

Disclosures: H.J. received research grants from Janssen Pharmaceutica and consulting fees with stock options from Guangzhou Burning Rock Medical Examination Institute Co, Ltd.
} 
Table 1 Distinct Features between Lung Adenocarcinoma and Squamous Cell Carcinoma

\begin{tabular}{|c|c|c|}
\hline Variable & Adenocarcinoma & Squamous cell carcinoma \\
\hline Location & Distal bronchioles $^{2}$ & Proximal airways ${ }^{3}$ \\
\hline Cell of origin & Type II pneumocytes, club cells ${ }^{2}$ & Basal cells ${ }^{3}$ \\
\hline Pathology & $\begin{array}{l}\text { Glandular differentiation with acini, tubules, } \\
\text { papillary structures }\end{array}$ & Keratin pearl, intracellular bridges ${ }^{3}$ \\
\hline Oncogenic drivers & $\begin{array}{l}\text { KRAS mut, EGFR mut, } H E R 2 \text { mut, } B R A F \text { mut, } \\
\quad A L K \text { fusion, ROS fusion, RET fusion }{ }^{4-8}\end{array}$ & $\begin{array}{l}\text { FGFR amp/mut/fusion, DDR2 mut, } \\
\text { TRA2B-DNAH5 fusion }\end{array}$ \\
\hline
\end{tabular}

amp, amplification; mut, mutation.

well as specific biomarkers. ${ }^{4,5}$ For example, lung ADCs commonly express thyroid transcription factor-1 (TTF1), ${ }^{6}$ a tissue-specific transcription factor important for cell differentiation and lineage determination. In contrast, lung SCCs preferentially express p63, a p53-homologous nuclear protein mainly involved in stem cell commitment. Besides, lung ADCs and SCCs have different spectrum of oncogenic drivers. ${ }^{8-10}$ For example, activating mutations of epidermal growth factor receptor $(E G F R)$, or anaplastic lymphoma kinase $(A L K)$ fusions, or $R E T$ fusions are mainly found in lung ADCs, whereas $F G F R$ amplification, $D D R 2$ mutations, and TRA2B-DNAH5 fusion are preferentially detected in SCCs. ${ }^{10-14}$

Interestingly, lung tumors with mixed adenomatous and squamous pathologies are consistently detected in the clinic. These tumors are clinically termed as adenosquamous cell carcinoma (Ad-SCC) when both pathological components exceeds $10 \%{ }^{2}$ In comparison to either ADCs or SCCs, lung Ad-SCC remains as the most deadly disease with worse prognosis. ${ }^{15}$ The consistent clinical observation of lung Ad-SCC leads to the attractive hypothesis about potential phenotypic transition between ADC and SCC. In 1981, Steele and Nettesheim ${ }^{16}$ designed an elegant experiment to clarify the monoclonal origin of this specific subtype of NSCLC. They dissected rat lung Ad-SCC specimens and established single-cell clones for subcutaneous inoculation into mice. Interestingly, pathological analysis showed that these single-cell clones could produce a variety of pathologies, including lung ADC, SCC, and Ad-SCC individually. ${ }^{16}$ This indicates a strong plasticity of lung Ad-SCC. In 1999, through clonal analyses in four human lung Ad-SCC, Niho et $\mathrm{a}^{17}$ found that both adenomatous and squamous components consistently showed the monoclonal origin. This has been further supported by multiple studies of gene mutational spectra analyses later on. For example, the mutations of EGFR or Kirsten rat sarcoma viral oncogene homolog $(K R A S)$ are frequently detected to be identical in adenomatous and squamous components of human lung Ad-SCC. ${ }^{18-20}$ Similar to ADCs, lung Ad-SCCs are mainly located at distal airways. ${ }^{21,22}$ Moreover, Ad-SCCs share similar oncogenic driver spectrum with ADC but not with SCC, ${ }^{18}$ indicative of the ADC to SCC transdifferentiation (AST) rather than the reverse transition. In fact, accumulating evidence from mice experiments and clinical observations convincingly support the AST. In this review, we will summarize recent progress on AST and raise some challenging questions, with the hope to better understand the pathogenesis of lung Ad-SCC and provide novel therapeutic strategies for this deadly disease.

\section{In Vivo Evidence in Supporting the ADC to SCC Transdifferentiation}

Despite several lines of clinical evidence implicating the existence of AST, there is still lack of direct proof. In the clinic, it remains technically difficult to obtain sequential biopsy specimens from a single patient because of ethical issues. Even with the availability of multiple biopsy specimens, the high intratumoral heterogeneity dampens the reach of conclusive point for AST. In contrast, the genetically engineered mouse model (GEMM) might serve as the ideal system for investigating this potential phenotypic transition. Recent studies of various GEMMs have tremendously improved our understanding of cancer initiation, progression, and metastasis. Undoubtedly, the GEMM is a useful platform to dissect molecular and cellular mechanisms of lung tumorigenesis. With specific gene mutations, GEMM closely recapitulates the molecular and cellular events of human lung cancer. Recent studies based on Lkbl-deficient GEMMs have provided convincing evidence to support the existence of AST in vivo.

Serine-threonine kinase 11 [STK11; alias liver kinase B1 $(L K B 1)]$, is a ubiquitously expressed gene. LKB1 can activate multiple downstream serine-threonine kinases through phosphorylation of a conserved threonine in the T-loop of the kinase domain, such as AMP-activated protein kinase and microtubule affinity regulating kinase. ${ }^{23,24}$ Germline inactivating mutations of $L K B 1$ have been reported to underlie the familial Peutz-Jeghers syndrome characterized by hamartomatous polyps. ${ }^{25,26}$ Recent studies have revealed an essential tumor-suppressive role of LKB1 in various neoplasms. ${ }^{27}$ In particular, somatic loss-of-function mutations of $L K B 1$ are frequently observed in various NSCLC subtypes, including ADC, SCC, and Ad-SCC. ${ }^{28}$ Interestingly, $L K B 1$ mutations are reported to be concurrent with KRAS 
A KL

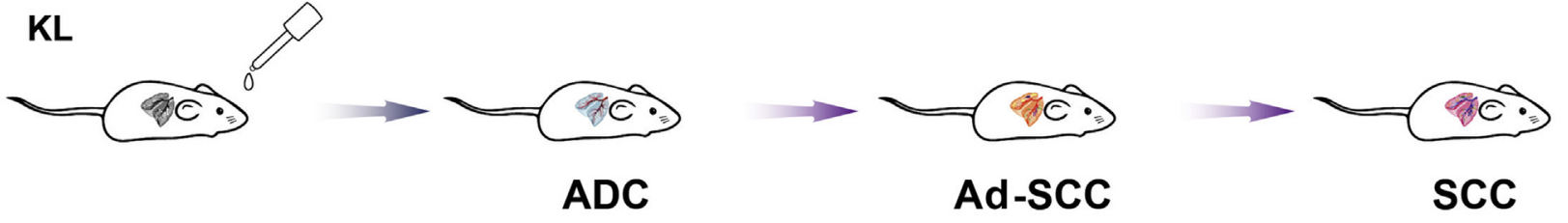

B
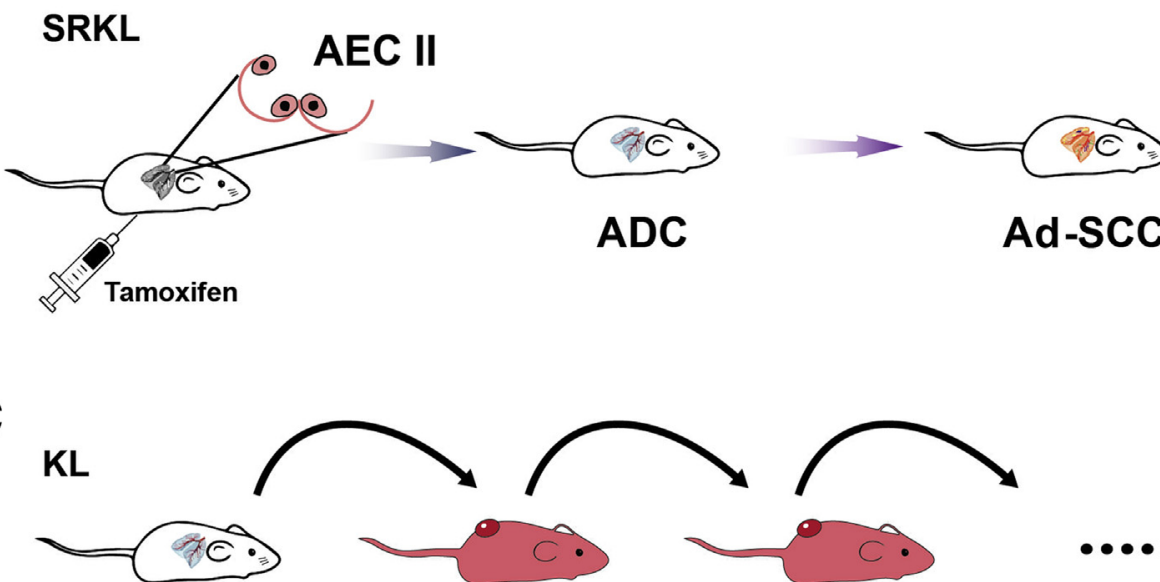

Ad-SCC

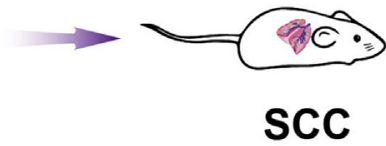

C
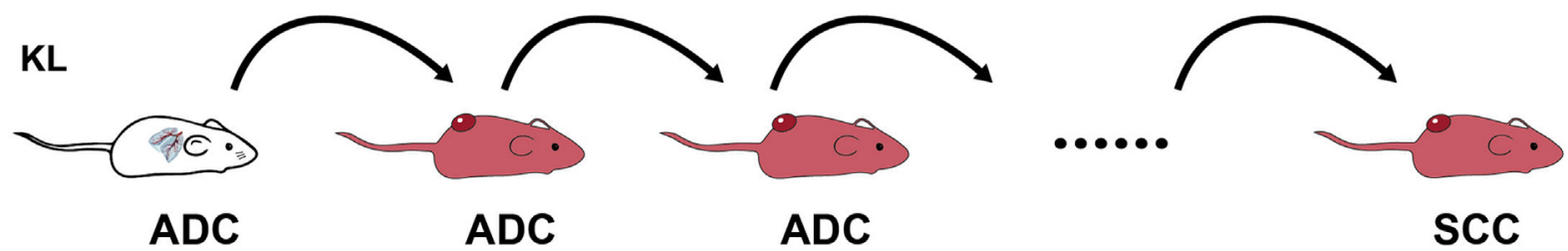

Figure 1 In vivo evidence from three mouse models supports lung adenocarcinoma (ADC) to squamous cell carcinoma (SCC) transdifferentiation. Illustration of the pathological transition from lung ADC to SCC in three different animal models, including Kras/Lkb1 (KL) Ad-Cre mouse model (A), surfactant protein C-CreERT2/Rosa26R-LacZ/Kras/Lkb1 (SRKL) mouse model (B), and the allograft model by serial transplantation of KL lung ADC into nude mice s.c. (C). Ad-SCC, adenosquamous cell carcinoma; AEC, alveolar epithelial type II cell.

mutation. ${ }^{29}$ Mouse modeling of this specific NSCLC subtype has resulted in certain interesting findings. For example, the $K r a s^{\mathrm{G} 12 \mathrm{D}} / L k b 1^{\mathrm{L} / \mathrm{L}}(K L)$ mouse model with AdCre nasal inhalation, which triggers concurrent deletion of $L k b 1$ and activation of $\operatorname{Kras}^{G 12 D}$ expression, shows accelerated malignant progression and metastasis as well as a wide pathological spectrum, including lung ADC and SCC (Figure 1A). ${ }^{30}$ Several early studies have uncovered the molecular mechanisms responsible for lung ADC metastases triggered by $L k b 1$ loss. It is now known that $L k b 1$ loss can activate the mammalian target of rapamycin-hypoxia inducible factor $1 \alpha$ axis and down-stream expression of lysyl oxidase (LOX). An increased LOX level can promote excessive collagen deposition and initiate extracellular matrix (ECM) remodeling, which, in turn, triggers integrin signaling, including SRC activation and cancer metastasis. ${ }^{30,31}$ Besides, studies also show that $L k b l$ loss can upregulate NEDD9 expression via the salt-inducible kinase 2-cAMP response element binding protein axis, which induces the epithelial-mesenchymal transition through focal adhesion kinase activation and thus promotes lung cancer metastasis. $^{30,32}$ These cell autonomous and non-cell autonomous signals triggered by $L k b 1$ loss might work synergistically to promote lung cancer metastasis. ${ }^{30,32}$

How the SCCs develop in KL Ad-Cre mouse model remains as an intriguing question. Detailed pathological and molecular studies have provided multiple layers of evidence in supporting the transition from ADC to SCC..$^{33-35}$ First, lung ADC in $K L$ model clearly show the continuously progressive pattern from atypical adenomatous hyperplasia or epithelial hyperplasia, to adenoma, and eventually to ADC. In contrast, SCCs are detectable only at late stage without preceding lesions, such as squamous metaplasia. Second, the number of lung ADCs decreases with the appearance of SCC, indicating the potential transition from ADC to SCC. Strikingly, those nascent SCCs have bigger tumor volume than most ADCs. Moreover, most of nascent SCCs exhibit mixed adenomatous and squamous pathologies, indicating that these pathologically mixed lung tumors, so-called mouse Ad-SCC, might represent as the intermediate stage for AST. Consistently, the percentage of lung Ad-SCC decreases while the pure SCC increases with time. ${ }^{33}$ In addition, most nascent SCCs express both ADC and SCC biomarkers, such as p63 and TTF1, further supporting the transdifferentiation from ADC to SCC.

Studies based on lineage-defined mouse model further support the transdifferentiation from lung ADC to SCC when LKB1 is deleted. Type II pnemocyte is commonly considered as the cell of origin for lung ADC. The transgenic mouse allele surfactant protein $\mathrm{C}-\mathrm{CreERT} 2$ could specifically restrict the CreERT2 expression in type II pnemocyte and produce lung ADC only when crossed with $\operatorname{Kras}^{G 12 D}$ mice. Interestingly, when crossed to $K L$ mouse model with reporter allele Rosa-Loxp-Stop-Loxp-LacZ, the compound lung cancer mouse model surfactant protein C-CreERT2/Rosa-Loxp-Stop-Loxp-LacZ/Kras ${ }^{\mathrm{G} 12 \mathrm{D}} / \mathrm{Lkbl}^{\mathrm{L} / \mathrm{L}}$ 
(SRKL) display both lung ADC and SCC when treated with tamoxifen to activate the Cre-ERT2 (Figure 1B). The tumor progression pattern and unique features of SCC from SRKL model resemble those observed in $K L$ Ad-Cre mouse model, further supporting the potential transdifferentiation from lung ADC to SCC. More important, lineage tracing analyses of the LacZ reporter confirm that lung SCC in SRKL model are derived from type II pnemocyte, the cell of origin for lung ADC. ${ }^{33}$ Because no early lesion for SCC is detectable, these data support that the lung SCC in SRKL mouse model is transdifferentiated from ADC.

The phenotypic transition from ADC to SCC is further supported by the serial transplantation of freshly dissected Lkb1-deficient mouse lung ADC in immunodeficient mice (Figure 1C). At early passages, these allograft tumors consistently show adenomatous pathology, whereas at late passages approximately $30 \%$ tumors are found to display squamous pathology. ${ }^{33}$ Moreover, these squamous lesions display strong p63 nuclear staining, ${ }^{33}$ further supporting the squamous transition.

Consistent with these observations, Mukhopadhyay et $\mathrm{al}^{36}$ have recently found that the $\operatorname{SOX} 2^{\text {hi }} / \mathrm{LKB} 1^{\text {null }}$ mouse lung ADCs display the expression of squamous biomarkers, including p63, cytokeratin-5 (K5), and cytokeratin-14 (K14), indicative of an early squamous transdifferentiation. SOX2 is a crucial transcription factor expressed in a variety of embryonic and adult stem cells. ${ }^{37}$ Amplification of SOX2 has been frequently detected in human lung SCC. ${ }^{9}$ Recently, Ferone et $\mathrm{al}^{38}$ showed that SOX2 overexpression in $\mathrm{Pten}^{-/-} / \mathrm{Cdkn2ab^{-/- }}$ type II pnemocyte could produce lung SCC. Although detailed analyses of early lung tumor lesions are necessary, these data suggest that SOX2 might serve as an important determining switch in promoting lung SCC from different cell of origin.

Findings of AST from mouse studies are further supported by clinical investigation. Approximately $6 \%$ to $10 \%$ of human ADCs are found to express squamous biomarker p63. ${ }^{24,34,39}$ Recently, Rekhtman et $\mathrm{al}^{5}$ reported that approximately 3\% of SCCs express both ADC and SCC biomarkers, such as TTF1 and p63. Other study has also supported such double positivity for p63 and TTF1 in both lung ADC and SCC. ${ }^{40}$ Interestingly, we find that all of the lung SCCs, which are double positive for TTF1 and p63, show the inactivation of LKB $1,{ }^{34}$ indicative of the essential role of LKB 1 in regulating such phenotypic transition. Moreover, the double positivity for p63 and TTF1 is detectable in both adenomatous and squamous components of human lung Ad-SCC without LKB1 expression. ${ }^{34}$ These clinical observations further strengthen the findings of AST from mouse models.

\section{ECM Remodeling and Metabolic Reprogramming Orchestrate AST}

ECM is a complex network of protein and glycoprotein and plays essential roles in regulating development, wound healing, and tissue homeostasis. ${ }^{41}$ Abnormal ECM dynamics are involved in cancer malignant progression, including angiogenesis and metastasis. ${ }^{42}$ LOX, which catalyzes the final enzymatic reaction required for collagen cross-linking, is crucial for collagen deposition and stabilization of the ECM structural components. ${ }^{43}$ Aberrant LOX expression or enzymatic activity is detectable in various malignant neoplasms, including breast cancer and lung cancer. ${ }^{44-47}$ Lung SCCs from $K L$ mouse model show significant reduction of collagen deposition in comparison with ADCs. In line with this, LOX mRNA and protein levels remarkably decrease in lung SCCs. Ectopic expression of a dominant negative minicollagen, which significantly inhibits collagen deposition, can accelerate the AST in $K L$ model. ${ }^{33}$ Conversely, forced expression of LOX, which promotes excessive collagen deposition, significantly dampens the squamous transdifferentiation. In addition, treatments with $\beta$-aminoproprionitrile or D-penicillamine, which either directly inhibits LOX enzymatic activity or neutralizes the aldehydes generated by LOX enzymatic reaction, could minimize collagen deposition and thus promote the AST. ${ }^{33}$ These data together demonstrate that ECM remodeling involving decreased LOX expression and collagen deposition in $K L$ mouse model functionally regulate the ADC to SCC transdifferentiation.

ECM depletion promotes cytosolic localization of Yesassociated protein (YAP) and thus inhibits the formation and activation of YAP/TEAD transcription factor complex, the main downstream effector of Hippo pathway. ${ }^{35}$ YAP is well documented to promote cell proliferation and fate determination. ${ }^{48,49}$ Aberrant YAP expression and nuclear localization are frequently observed in various epithelia cancers, such as lung, colorectal, ovarian, and prostate cancers. ${ }^{49-53}$ However, YAP is significantly inactivated in lung SCCs versus ADCs in $K L$ mouse model, indicating a potentially unexpected role in AST. In supporting this, forced YAP activation significantly inhibits the AST, whereas knockdown of YAP conversely accelerates the AST. In mouse lung ADC, YAP activation could upregulate ZEB2 expression, which intrinsically represses TP63 transcription. This might help maintain the lineage commitment of lung ADC. However, during the ADC to SCC transdifferentiation, YAP is significantly inactivated because of ECM depletion, which, in turn, down-regulates ZEB2 level and lifts up the intrinsic suppression of TP63 transcription (Figure 2). Increased p63 expression is capable to drive downstream squamous signature gene expression and eventually contributes to squamous transdifferentiation.

Our previous studies have shown that both LOX and NEDD9 expression are up-regulated by $L k b 1$ loss in mouse lung ADC. ${ }^{54}$ In contrast, the expression of these two key molecules is significantly down-regulated in lung SCC during AST. Thus, how the signals are reversed in lung SCC remains as an intriguing question. We find that during malignant progression of lung $\mathrm{ADC}$, the pentose phosphate pathway is deregulated because of the limited nutrient and aggravates reactive oxygen species (ROS) accumulation (Figure 2). 


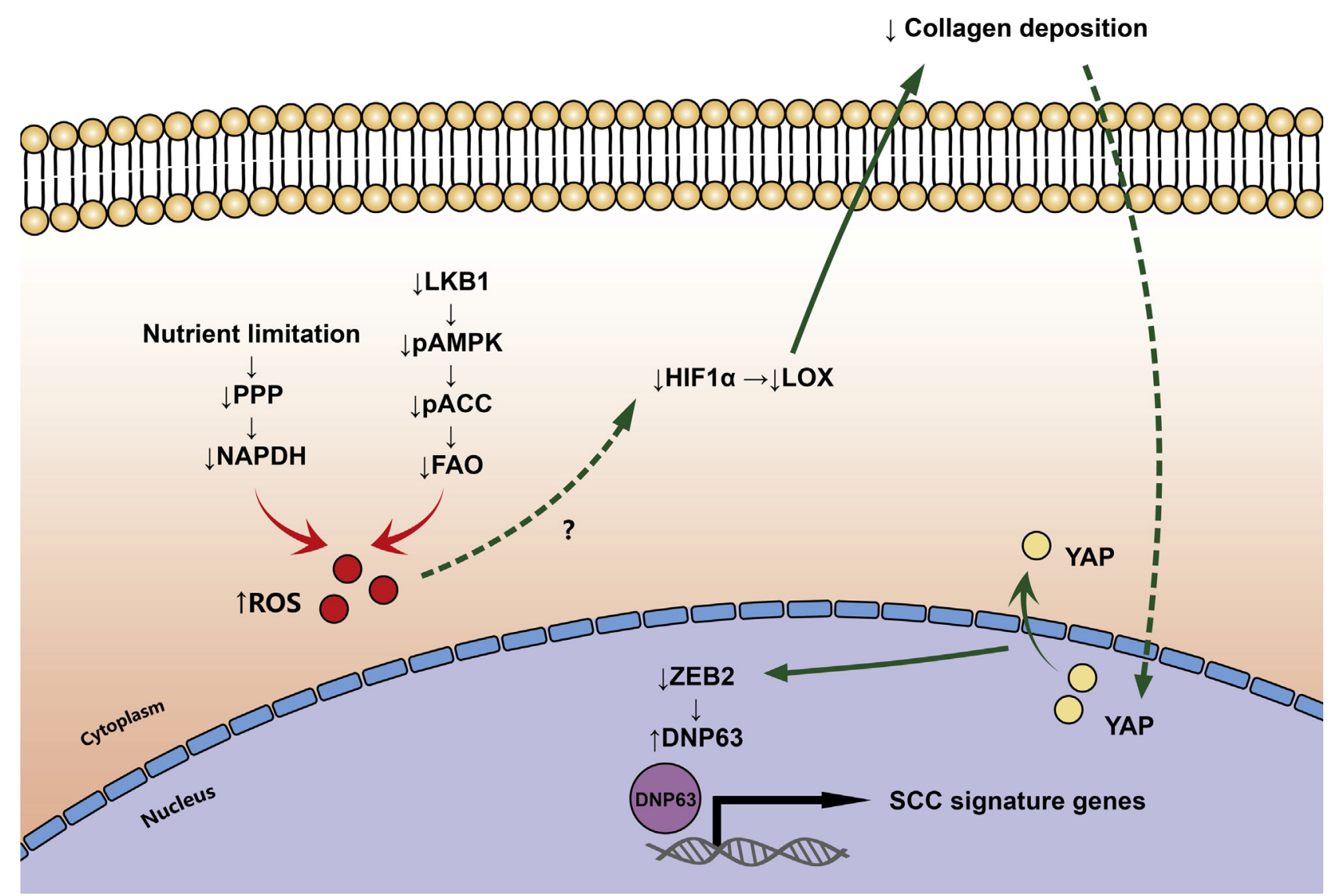

Figure 2 Mechanistic insights into adenocarcinoma to squamous cell carcinoma (SCC) transdifferentiation (AST). During AST in $K L$ mouse model, reactive oxygen species (ROS) levels in lung ADC increase because of decreased NADPH level and deregulated pentose phosphate pathway (PPP) under nutrient limitation accompanied with tumor malignant progression. Meanwhile, $L k b 1$ loss results in defective activation of downstream signaling, especially the AMPactivated protein kinase (AMPK)-acetyl-coenzyme A carboxylase axis and the fatty acid oxidation (FA0) pathway, and thus causes ineffective ROS clearance. Through an unknown mechanism, this uncontrolled ROS accumulation reverses the signal pathways initially activated by $L k b 1$ loss, including the hypoxia inducible factor (HIF) $1 \alpha$-lysyl oxidase (LOX) axis. Decreased LOX expression leads to collagen depletion, which causes Yes-associated protein (YAP) phosphorylation and cytosolic localization. YAP inactivation, in turn, results in ZEB2 down-regulation and lifts up the intrinsic suppression of TP63 transcription, and thus promotes squamous signature gene expression and eventually squamous transdifferentiation. Question marks indicate that the regulatory relationship is not quite clear, or no direct evidence.

Meanwhile, Lkbl loss results in defective activation of downstream signaling, especially the AMP-activated protein kinase-acetyl-coenzyme A carboxylase axis and the fatty acid oxidation pathway, and thus causes ineffective ROS clearance. These two effects work together to promote uncontrolled excessive ROS accumulation. Through an unknown mechanism, the oxidative stress reverses the signal pathways initially activated by $L k b l$ deficiency, including the hypoxia inducible factor $1 \alpha-\mathrm{LOX}$ axis and triggered ECM remodeling, which resulted in collagen depletion, YAP/ZEB inactivation, p63 up-regulation, and eventually squamous transdifferentiation (Figure 2).

\section{AST as a Novel Cellular Mechanism for Drug Resistance}

Mechanistic studies about AST in $K L$ mouse model also provide novel insights into drug resistance. When treated with
LOX inhibitors, mouse lung SCCs show strong resistance, whereas $L k b l$-deficient lung ADCs regress significantly with decreased cell proliferation and increased apoptosis. ${ }^{33}$ Similarly, phenformin or piperlongumine treatment, which significantly promotes the regression of mouse lung ADC mainly through promoting ROS production, shows almost no effect on lung SCC in $K L$ mouse model ${ }^{33,34}$ (Figure 3). These data from animal models suggest that AST might represent as a novel mechanism for drug resistance.

This is further supported by recent clinical observations (Figure 3). Most lung ADC patients with oncogenic EGFR mutations, such as L858R and exon 19 deletion, are initially sensitive to tyrosine kinase inhibitor treatments. However, drug resistance often occurs within 6 months to 1 year of tyrosine kinase inhibitor treatments. ${ }^{55,56}$ Multiple molecular mechanisms for tyrosine kinase inhibitor resistance have been reported, including genetic alterations such as T790M mutation or MET amplification, alternative pathway activation, and phenotypic transition such as 


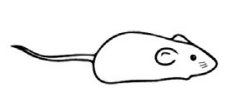

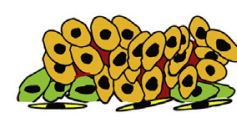

ADC

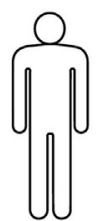

ADC
DPA/BAPN/PL/Phen
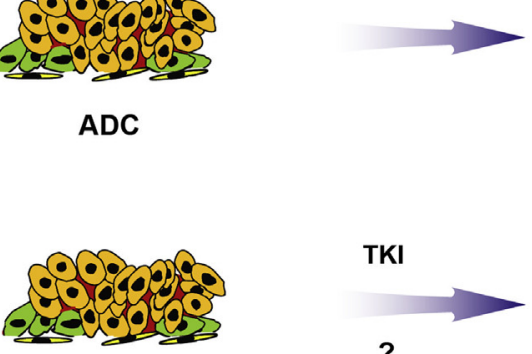

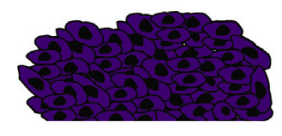

SCC

Sensitive

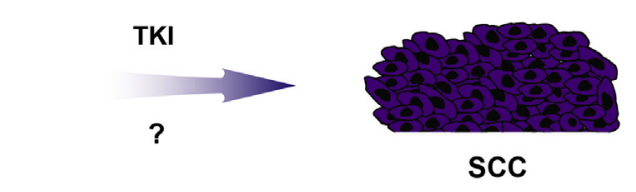

Resistant
Figure 3 Transdifferentiation from lung adenocarcinoma (ADC) to squamous cell carcinoma (SCC) represents as a novel cellular mechanism for drug resistance. The ADC to SCC transdifferentiation (AST) in mouse models confers lung tumor with strong capabilities to resist the treatments of small molecular compounds, including D-penicillamine (DPA), $\beta$-aminoproprionitrile (BAPN), piperlongumine (PL), and phenformin (Phen). Similarly, the potential AST observed in relapsed lung adenocarcinoma patients with EGFR mutations indicates drug resistance toward tyrosine kinase inhibitor (TKI). Question mark indicates that the proof of AST during TKI resistance is not quite strong; more clinical samples are needed to verify this hypothesis. epithelial-mesenchymal transition and SCLC transformation. ${ }^{57,58}$ Interestingly, several recent studies reveal that relapsed lung ADC patients sometimes display squamous pathology on second biopsies; and consistently, these SCCs show identical EGFR mutations as initially identified in lung ADC. ${ }^{59-65}$ Another relapsed lung ADC patient shows SCC pathology in his or her brain metastasis. ${ }^{66}$ Such squamous transdifferentiation also occurs during chemoresistance development, suggesting that AST might represent as a common mechanism for drug resistance. The original lung cancer might be misdiagnosed as ADC but contain squamous components, potentially because of small biopsy specimens. In considering the single-cell origin and clonal evolution of lung cancer, the observation of mixed adenomatous and squamous components in a single lesion does support the occurrence of AST. This AST process, occurring either before or after drug treatment, clearly confers these tumors with drug resistance.

\section{Perspectives}

Phenotypic transition, such as AST, reflects strong plasticity of lung cancer. Integrative studies of murine models and human clinical specimens have convincingly corroborated the existence of AST. Moreover, emerging data have indicated that AST might serve as a novel mechanism for drug resistance, which holds important clinical implication. Nonetheless, the field of AST is still in its infancy. Many important and challenging questions remain to be answered.

GEMM study shows that $L k b l$-deficient lung ADCs progressively transdifferentiate to SCC, implicating an essential role of LKB1 in AST. Although this is further supported by clinical observation of LKB1 inactivation in lung Ad-SCC, the mutation rate of $L K B 1$ in NSCLC is $<30 \%$ and most lung cancer still remains as $L K B 1$ wild type. Although epigenetic regulation or protein degradation could result in LKB1 inactivation, ${ }^{67}$ it remains interesting to ask whether an LKB1-independent mechanism contributes to AST. Systematic investigation into human lung Ad-SCC using genomic and proteomic methods might help identify essential factors regulating the AST process. This will definitely help establish new mouse models for AST studies.

Clinical evidence for AST needs to be further strengthened. Up to now, only 11 cases of potential AST have been reported worldwide. ${ }^{59-66}$ With current development of effective targeted therapies for lung cancer patients, the repetitive biopsy specimens are becoming more and more popular. Investigation into a large body of biopsy specimens will certainly provide more evidence to support AST as well as its potential link with drug resistance. With this, it will be also interesting to explore how LKB1 inactivation affects drug resistance in those lung cancer patients with potential AST.

The patient-derived xenograft model might serve as another helpful platform to study AST. In contrast to traditional xenograft model, patient-derived xenograft models show its unique advantage with largely preserved tumor heterogeneity and microenvironment. It remains interesting to ask if AST does happen in certain patientderived xenograft models with drug treatments. However, this could be challenging. First, AST might only occur in certain lung tumors with specific genetic alteration combination; second, it remains unclear whether these genetic alterations provide advantage for patient-derived xenograft establishment; last, it is difficult to identify therapeutic strategies suitable for AST.

Current studies have demonstrated the essential role of the ECM remodeling in AST. In contrast to ADC, mouse lung SCCs exhibit decreased expression of LOX as well as other LOX family members, such as LOXL2. The Lox family contains Lox, and Lox-like 1-4 (Loxl1-4), ${ }^{68}$ which participates in covalent intramolecular or intermolecular cross-linking of collagen and promotes collagen deposition and extracellular matrix assembly. Although previous studies have indicated strong cancer-promotive function of LOX and LOXL2, ${ }^{69-72}$ LOX inhibition via $\beta$-aminoproprionitrile or D-penicillamine significantly promotes the ADC to SCC transdifferentiation as well as 
drug resistance. We reason this phenomenon might be specific to SCC pathology. SCC seems more dependent on cell junctions rather than extracellular matrix for cell proliferation and survival. Indeed, many genes associated with cell junctions, such as desmosome-associated genes, ${ }^{33,73,74}$ are robustly up-regulated in SCC relative to ADC. Future work will be necessary to clarify the potential involvement of other LOX family members and desmosome-related genes in AST.

In addition, aberrant ROS accumulation is now known to promote lung AST. Because of LKB1 inactivation, downstream pathways, especially the fatty acid oxidation pathway, could not be effectively activated during lung ADC malignant progression, which results in defective ROS elimination and thus uncontrolled oxidative stress accumulation. It remains interesting to study how lung tumors resist such high oxidative stress during AST. Mechanistic insights into AST process will certainly provide a base for future development of effective therapeutic strategies to overcome drug resistance associated with AST.

\section{Acknowledgments}

We thank all members of Ji Laboratory for helpful discussion.

\section{References}

1. Edwards BK, Noone AM, Mariotto AB, Simard EP, Boscoe FP, Henley SJ, Jemal A, Cho H, Anderson RN, Kohler BA, Eheman CR, Ward EM: Annual Report to the Nation on the status of cancer, 1975-2010, featuring prevalence of comorbidity and impact on survival among persons with lung, colorectal, breast, or prostate cancer. Cancer 2014, 120:1290-1314

2. National Research Council. (U.S.) Committee on Pathology, Universities Associated for Research and Education in Pathology, Armed Forces Institute of Pathology (U.S.), American Cancer Society, Colby TV, Koss MN, Travis WD: Tumors of the Lower Respiratory Tract, 3rd series. Washington, DC, Armed Forces Institute of Pathology, 1995

3. Sutherland KD, Berns A: Cell of origin of lung cancer. Mol Oncol 2010, 4:397-403

4. Fujii T, Dracheva T, Player A, Chacko S, Clifford R, Strausberg RL, Buetow K, Azumi N, Travis WD, Jen J: A preliminary transcriptome map of non-small cell lung cancer. Cancer Res 2002, 62:3340-3346

5. Rekhtman N, Ang DC, Sima CS, Travis WD, Moreira AL: Immunohistochemical algorithm for differentiation of lung adenocarcinoma and squamous cell carcinoma based on large series of whole-tissue sections with validation in small specimens. Mod Pathol 2011, 24: $1348-1359$

6. Wu M, Wang B, Gil J, Sabo E, Miller L, Gan L, Burstein DE: p63 and TTF-1 immunostaining. Am J Clin Pathol 2003, 119:696-702

7. Wang BY, Gil J, Kaufman D, Gan L, Kohtz DS, Burstein DE: p63 in pulmonary epithelium, pulmonary squamous neoplasms, and other pulmonary tumors. Hum Pathol 2002, 33:921-926

8. Cancer Genome Atlas Research Network: Comprehensive molecular profiling of lung adenocarcinoma. Nature 2014, 511:543-550

9. Cancer Genome Atlas Research Network: Comprehensive genomic characterization of squamous cell lung cancers. Nature 2012, 489: $519-525$
10. Li F, Fang Z, Zhang J, Li C, Liu H, Xia J, Zhu H, Guo C, Qin Z, Li F: Identification of TRA2B-DNAH5 fusion as a novel oncogenic driver in human lung squamous cell carcinoma. Cell Res 2016, 26: $1149-1164$

11. Sugio K, Uramoto H, Ono K, Oyama T, Hanagiri T, Sugaya M, Ichiki Y, So T, Nakata S, Morita M, Yasumoto K: Mutations within the tyrosine kinase domain of EGFR gene specifically occur in lung adenocarcinoma patients with a low exposure of tobacco smoking. $\mathrm{Br}$ J Cancer 2006, 94:896-903

12. Rekhtman N, Paik PK, Arcila ME, Tafe LJ, Oxnard GR, Moreira AL, Travis WD, Zakowski MF, Kris MG, Ladanyi M: Clarifying the spectrum of driver oncogene mutations in biomarker-verified squamous carcinoma of lung: lack of EGFR/KRAS and presence of PIK3CA/AKT1 mutations. Clin Cancer Res 2012, 18:1167-1176

13. Li F, Feng Y, Fang R, Fang Z, Xia J, Han X, Liu X-Y, Chen H, Liu H, Ji H: Identification of RET gene fusion by exon array analyses in "pan-negative" lung cancer from never smokers. Cell Res 2012, 22:928

14. Wang R, Hu H, Pan Y, Li Y, Ye T, Li C, Luo X, Wang L, Li H, Zhang Y: RET fusions define a unique molecular and clinicopathologic subtype of non-small-cell lung cancer. J Clin Oncol 2012, 30: 4352-4359

15. Nakagawa K, Yasumitu T, Fukuhara K, Shiono H, Kikui M: Poor prognosis after lung resection for patients with adenosquamous carcinoma of the lung. Ann Thorac Surg 2003, 75:1740-1744

16. Steele VE, Nettesheim P: Unstable cellular differentiation in adenosquamous cell carcinoma. J Natl Cancer Inst 1981, 67:149-154

17. Niho S, Yokose T, Kodama T, Nishiwaki Y, Mukai K: Clonal analysis of adenosquamous carcinoma of the lung. Jpn J Cancer Res 1999, 90:1244-1247

18. Kang SM, Kang HJ, Shin JH, Kim H, Shin DH, Kim SK, Kim JH, Chung KY, Kim SK, Chang J: Identical epidermal growth factor receptor mutations in adenocarcinomatous and squamous cell carcinomatous components of adenosquamous carcinoma of the lung. Cancer 2007, 109:581-587

19. Jia XL, Chen G: EGFR and KRAS mutations in Chinese patients with adenosquamous carcinoma of the lung. Lung Cancer 2011, 74: 396-400

20. Tochigi N, Dacic S, Nikiforova M, Cieply KM, Yousem SA: Adenosquamous carcinoma of the lung: a microdissection study of KRAS and EGFR mutational and amplification status in a western patient population. Am J Clin Pathol 2011, 135:783-789

21. Lee Y, Chung JH, Kim SE, Kim TJ, Lee KW: Adenosquamous carcinoma of the lung: CT, FDG PET, and clinicopathologic findings. Clin Nucl Med 2014, 39:107-112

22. Moon Y, Lee KY, Sung SW, Park JK: Differing histopathology and prognosis in pulmonary adenocarcinoma at central and peripheral locations. J Thorac Dis 2016, 8:169-177

23. Katajisto P, Vallenius T, Vaahtomeri K, Ekman N, Udd L, Tiainen M, Mäkelä TP: The LKB1 tumor suppressor kinase in human disease. Biochim Biophys Acta 2007, 1775:63-75

24. Argon A, Nart D, Veral A: The value of cytokeratin 5/6, p63 and thyroid transcription factor- 1 in adenocarcinoma, squamous cell carcinoma and non-small-cell lung cancer of the lung. Turk Patoloji Derg 2015, 31:81-88

25. Hemminki A: The molecular basis and clinical aspects of Peutz Jeghers syndrome. Cell Mol Life Sci 1999, 55:735-750

26. Jenne DE, Reomann H, Nezu J, Friedel W, Loff S, Jeschke R, Müller O, Back W, Zimmer M: Peutz-Jeghers syndrome is caused by mutations in a novel serine threonine kinase. Nat Genet 1998, 18: $38-43$

27. Hardie DG: The LKB1-AMPK pathway-friend or foe in cancer? Cancer Cell 2013, 23:131-132

28. Makowski L, Hayes DN: Role of LKB1 in lung cancer development. Br J Cancer 2008, 99:683-688

29. Sanchez-Cespedes M, Parrella P, Esteller M, Nomoto S, Trink B, Engles JM, Westra WH, Herman JG, Sidransky D: Inactivation of 
LKB1/STK11 is a common event in adenocarcinomas of the lung. Cancer Res 2002, 62:3659-3662

30. Ji H, Ramsey MR, Hayes DN, Fan C, McNamara K, Kozlowski P, Torrice C, Wu MC, Shimamura T, Perera SA, Liang MC, Cai D, Naumov GN, Bao L, Contreras CM, Li D, Chen L, Krishnamurthy J, Koivunen J, Chirieac LR, Padera RF, Bronson RT, Lindeman NI, Christiani DC, Lin X, Shapiro GI, Janne PA, Johnson BE, Meyerson M, Kwiatkowski DJ, Castrillon DH, Bardeesy N, Sharpless NE, Wong KK: LKB1 modulates lung cancer differentiation and metastasis. Nature 2007, 448:807-810

31. Carretero J, Shimamura T, Rikova K, Jackson AL, Wilkerson MD, Borgman CL, Buttarazzi MS, Sanofsky BA, McNamara KL, Brandstetter KA, Walton ZE, Gu TL, Silva JC, Crosby K, Shapiro GI, Maira SM, Ji H, Castrillon DH, Kim CF, Garcia-Echeverria C, Bardeesy N, Sharpless NE, Hayes ND, Kim WY, Engelman JA, Wong KK: Integrative genomic and proteomic analyses identify targets for Lkb1-deficient metastatic lung tumors. Cancer Cell 2010, 17:547-559

32. Gao Y, Xiao Q, Ma H, Li L, Liu J, Feng Y, Fang Z, Wu J, Han X, Zhang J, Sun Y, Wu G, Padera R, Chen H, Wong KK, Ge G, Ji H: LKB1 inhibits lung cancer progression through lysyl oxidase and extracellular matrix remodeling. Proc Natl Acad Sci U S A 2010, 107 : 18892-18897

33. Han X, Li F, Fang Z, Gao Y, Li F, Fang R, Yao S, Sun Y, Li L, Zhang W, Ma H, Xiao Q, Ge G, Fang J, Wang H, Zhang L, Wong KK, Chen H, Hou Y, Ji H: Transdifferentiation of lung adenocarcinoma in mice with Lkb1 deficiency to squamous cell carcinoma. Nat Commun 2014, 5:3261

34. Li F, Han X, Li F, Wang R, Wang H, Gao Y, Wang X, Fang Z, Zhang W, Yao S, Tong X, Wang Y, Feng Y, Sun Y, Li Y, Wong KK, Zhai Q, Chen H, Ji H: LKB1 inactivation elicits a redox imbalance to modulate non-small cell lung cancer plasticity and therapeutic response. Cancer Cell 2015, 27:698-711

35. Gao Y, Zhang W, Han X, Li F, Wang X, Wang R, Fang Z, Tong X, Yao S, Li F, Feng Y, Sun Y, Hou Y, Yang Z, Guan K, Chen H, Zhang L, Ji H: YAP inhibits squamous transdifferentiation of Lkb1deficient lung adenocarcinoma through ZEB2-dependent DNp63 repression. Nat Commun 2014, 5:4629

36. Mukhopadhyay A, Berrett KC, Kc U, Clair PM, Pop SM, Carr SR, Witt BL, Oliver TG: Sox2 cooperates with Lkb1 loss in a mouse model of squamous cell lung cancer. Cell Rep 2014, 8:40-49

37. Sarkar A, Hochedlinger K: The sox family of transcription factors: versatile regulators of stem and progenitor cell fate. Cell Stem Cell 2013, 12:15-30

38. Ferone G, Song JY, Sutherland KD, Bhaskaran R, Monkhorst K, Lambooij JP, Proost N, Gargiulo G, Berns A: SOX2 is the determining oncogenic switch in promoting lung squamous cell carcinoma from different cells of origin. Cancer Cell 2016, 30:519-532

39. Mukhopadhyay S, Katzenstein AL: Subclassification of non-small cell lung carcinomas lacking morphologic differentiation on biopsy specimens: utility of an immunohistochemical panel containing TTF-1, napsin A, p63, and CK5/6. Am J Surg Pathol 2011, $35: 15-25$

40. Kim MJ, Shin HC, Shin KC, Ro JY: Best immunohistochemical panel in distinguishing adenocarcinoma from squamous cell carcinoma of lung: tissue microarray assay in resected lung cancer specimens. Ann Diagn Pathol 2013, 17:85-90

41. Bonnans C, Chou J, Werb Z: Remodelling the extracellular matrix in development and disease. Nat Rev Mol Cell Biol 2014, 15:786-801

42. Lu P, Weaver VM, Werb Z: The extracellular matrix: a dynamic niche in cancer progression. J Cell Biol 2012, 196:395-406

43. Kagan HM, Li W: Lysyl oxidase: properties, specificity, and biological roles inside and outside of the cell. J Cell Biochem 2003, 88:660-672

44. Erler JT, Bennewith KL, Nicolau M, Dornhöfer N, Kong C, Le QT, Chi JTA, Jeffrey SS, Giaccia AJ: Lysyl oxidase is essential for hypoxia-induced metastasis. Nature 2006, 440:1222-1226
45. Kirschmann DA, Seftor EA, Fong SF, Nieva DR, Sullivan CM, Edwards EM, Sommer P, Csiszar K, Hendrix MJ: A molecular role for lysyl oxidase in breast cancer invasion. Cancer Res 2002, 62: $4478-4483$

46. Kirschmann DA, Seftor EA, Nieva DR, Mariano EA, Hendrix MJ: Differentially expressed genes associated with the metastatic phenotype in breast cancer. Breast Cancer Res Treat 1999, 55:125-134

47. Payne SL, Hendrix MJ, Kirschmann DA: Paradoxical roles for lysyl oxidases in cancer-a prospect. J Cell Biochem 2007, 101: $1338-1354$

48. Lian I, Kim J, Okazawa H, Zhao J, Zhao B, Yu J, Chinnaiyan A, Israel MA, Goldstein LS, Abujarour R: The role of YAP transcription coactivator in regulating stem cell self-renewal and differentiation. Genes Dev 2010, 24:1106-1118

49. Zhao B, Tumaneng K, Guan KL: The Hippo pathway in organ size control, tissue regeneration and stem cell self-renewal. Nat Cell Biol 2011, 13:877-883

50. Barry ER, Camargo FD: The Hippo superhighway: signaling crossroads converging on the Hippo/Yap pathway in stem cells and development. Curr Opin Cell Biol 2013, 25:247-253

51. Hiemer SE, Varelas X: Stem cell regulation by the Hippo pathway. Biochim Biophys Acta 2013, 1830:2323-2334

52. Wang Y, Dong Q, Zhang Q, Li Z, Wang E, Qiu X: Overexpression of yes-associated protein contributes to progression and poor prognosis of non-small-cell lung cancer. Cancer Sci 2010, 101:1279-1285

53. Xu MZ, Yao TJ, Lee NP, Ng IO, Chan YT, Zender L, Lowe SW, Poon RT, Luk JM: Yes-associated protein is an independent prognostic marker in hepatocellular carcinoma. Cancer 2009, 115: 4576-4585

54. Feng Y, Wang Y, Wang Z, Fang Z, Li F, Gao Y, Liu H, Xiao T, Li F, Zhou Y, Zhai Q, Liu X, Sun Y, Bardeesy N, Wong KK, Chen H, Xiong ZQ, Ji H: The CRTC1-NEDD9 signaling axis mediates lung cancer progression caused by LKB1 loss. Cancer Res 2012, 72: $6502-6511$

55. Santarpia M, Gil N, Rosell R: Strategies to overcome resistance to tyrosine kinase inhibitors in non-small-cell lung cancer. Expert Rev Clin Pharmacol 2015, 8:461-477

56. Riely GJ, Yu HA: EGFR: the paradigm of an oncogene-driven lung cancer. Clin Cancer Res 2015, 21:2221-2226

57. Carrera S, Buque A, Azkona E, Aresti U, Calvo B, Sancho A, Arruti M, Nuno M, Rubio I, de Lobera AR, Lopez C, Vivanco GL: Epidermal growth factor receptor tyrosine-kinase inhibitor treatment resistance in non-small cell lung cancer: biological basis and therapeutic strategies. Clin Transl Oncol 2014, 16:339-350

58. Huang L, Fu L: Mechanisms of resistance to EGFR tyrosine kinase inhibitors. Acta Pharm Sin B 2015, 5:390-401

59. Haratani K, Hayashi H, Watanabe S, Kaneda H, Yoshida T, Takeda M, Shimizu T, Nakagawa K: Two cases of EGFR mutationpositive lung adenocarcinoma that transformed into squamous cell carcinoma: successful treatment of one case with rociletinib. Ann Oncol 2016, 27:200-202

60. Hsieh MS, Jhuang JY, Hua SF, Chou YH: Histologic evolution from adenocarcinoma to squamous cell carcinoma after gefitinib treatment. Ann Thorac Surg 2015, 99:316-319

61. Levin PA, Mayer M, Hoskin S, Sailors J, Oliver DH, Gerber DE: Histologic transformation from adenocarcinoma to squamous cell carcinoma as a mechanism of resistance to EGFR inhibition. J Thorac Oncol 2015, 10:e86-e88

62. Scher KS, Saldivar JS, Fishbein M, Marchevsky A, Reckamp KL: EGFR-mutated lung cancer with T790M-acquired resistance in the brain and histologic transformation in the lung. J Natl Compr Canc Netw 2013, 11:1040-1044

63. Jukna A, Montanari G, Mengoli MC, Cavazza A, Covi M, Barbieri F, Bertolini F, Rossi G: Squamous cell carcinoma "transformation" concurrent with secondary T790M mutation in resistant EGFR-mutated adenocarcinomas. J Thorac Oncol 2016, 11:e49-e51 
64. Kuiper JL, Ronden MI, Becker A, Heideman DA, van Hengel P, Ylstra B, Thunnissen E, Smit EF: Transformation to a squamous cell carcinoma phenotype of an EGFR-mutated NSCLC patient after treatment with an EGFR-tyrosine kinase inhibitor. J Clin Pathol 2015, 68:320-321

65. Bugano DDG, Kalhor N, Zhang J, Neskey M, William WN Jr: Squamous-cell transformation in a patient with lung adenocarcinoma receiving erlotinib: co-occurrence with T790M mutation. Cancer Treat Res Commun 2015, 4:34-36

66. Burkart J, Shilo K, Zhao W, Ozkan E, Ajam A, Otterson GA: Metastatic squamous cell carcinoma component from an adenosquamous carcinoma of the lung with identical epidermal growth factor receptor mutations. Case Rep Pulmonol 2015, 2015:283875

67. Fang R, Zheng C, Sun Y, Han X, Gao B, Li C, Liu H, Wong KK, Liu XY, Chen $\mathrm{H}$, Ji H: Integrative genomic analysis reveals a high frequency of LKB1 genetic alteration in Chinese lung adenocarcinomas. J Thorac Oncol 2014, 9:254-258

68. Barker HE, Cox TR, Erler JT: The rationale for targeting the LOX family in cancer. Nat Rev Cancer 2012, 12:540-552

69. Zhan P, Shen XK, Qian Q, Zhu JP, Zhang Y, Xie HY, Xu CH, Hao KK, Hu W, Xia N: Down-regulation of lysyl oxidase-like 2
(LOXL2) is associated with disease progression in lung adenocarcinomas. Med Oncol 2012, 29:648-655

70. Cano A, Santamaría PG, Moreno-Bueno G: LOXL2 in epithelial cell plasticity and tumor progression. Future Oncol 2012, 8: $1095-1108$

71. Mizuno K, Seki N, Mataki H, Matsushita R, Kamikawaji K, Kumamoto T, Takagi K, Goto Y, Nishikawa R, Kato M: Tumorsuppressive microRNA-29 family inhibits cancer cell migration and invasion directly targeting LOXL2 in lung squamous cell carcinoma. Int J Oncol 2016, 48:450-460

72. Peinado H, Moreno-Bueno G, Hardisson D, Pérez-Gómez E, Santos V, Mendiola M, de Diego JI, Nistal M, Quintanilla M, Portillo F: Lysyl oxidase-like 2 as a new poor prognosis marker of squamous cell carcinomas. Cancer Res 2008, 68:4541-4550

73. Boelens MC, van den Berg A, Vogelzang I, Wesseling J, Postma DS, Timens W, Groen HJ: Differential expression and distribution of epithelial adhesion molecules in non-small cell lung cancer and normal bronchus. J Clin Pathol 2007, 60:608-614

74. Kuner R, Muley T, Meister M, Ruschhaupt M, Buness A, Xu EC, Schnabel P, Warth A, Poustka A, Sültmann H: Global gene expression analysis reveals specific patterns of cell junctions in non-small cell lung cancer subtypes. Lung Cancer 2009, 63:32-38 